(C) 1998 International Press

Adv. Theor. Math. Phys. 2 (1998) 399-411

\title{
Branes and Fundamental Groups
}

\author{
Rajesh Gopakumar ${ }^{a}$, Cumrun Vafa ${ }^{b}$ \\ ${ }^{a, b}$ Department of Physics \\ University of California \\ Santa Barbara, CA 93106 \\ ${ }^{b}$ Lyman Laboratory of Physics \\ Harvard University \\ Cambridge, MA 02138
}

\begin{abstract}
We consider bound states of D-branes wrapped around cycles with non-trivial fundamental groups of finite order. We find a new mechanism for binding $\mathrm{D}$-branes by turning on flat discrete abelian and non-abelian gauge fields on their worldvolume. As a concrete application we study type IIB in the background where an $S^{3} / G$ shrinks, where $G$ is a discrete subgroup of $S U(2)$ acting freely on $S^{3}$.
\end{abstract}

\section{Introduction}

One of the most beautiful aspects of our recent insight into non-perturbative aspects of string theory involves how branes wrapped around vanishing cycles can lead to new low energy degrees of freedom. The particular case of type IIB with a vanishing $S^{3}$ in particular leads to a massless hypermultiplet corresponding to a 3-brane wrapped around it [1]. Due to the identification of D-branes as the sources of RR charge [2] one can gain more insight into questions involving bound states of D-branes [3]. In particular one can show [4] that multiple wrappings of 3-branes around $S^{3}$ do not lead to any bound states, as anticipated in [1].

In this note we consider D-branes wrapped around cycles with non-trivial fundamental group $G$ of finite order. We will show that for each non-trivial 
irreducible representation of $G$ of dimension $d_{i}$, we obtain a new bound state of $d_{i} \mathrm{D}$-branes wrapped around the same cycle. The basic idea is to turn on a non-trivial discrete flat gauge field in the D-brane worldvolume. Note that this can be done due to the fact that for $d_{i}$ coincident $D$-branes we obtain a $U\left(d_{i}\right)$ gauge symmetry. The condition that the representation be irreducible gets translated to having bound states of D-branes. We illustrate the basic results in the context of type IIB compactifications on Calabi-Yau threefolds where an $S^{3} / G$ vanishes, where $G$ is a subgroup of $S U(2)$ freely acting on $S^{3}$. In particular, in this example we find that the well-known correspondence between discrete subgroups of $S U(2)$ and affine Dynkin diagrams is rather useful in giving the spectrum of bound states. Namely, for each subgroup of $S U(2)$ the number of bound states is equal to the number of nodes of the corresponding affine Dynkin diagram. Moreover the Dynkin index corresponding to each node signifies the number of D-branes of which this is a bound state.

\section{D-Branes and Non-Trivial Fundamental Group}

Let us consider type IIA/B strings compactified on some manifold $M$. Consider a compact submanifold $C \subset M$. Let us assume that it is a supersymmetric cycle. Moreover let us assume that it has a non-trivial fundamental group:

$$
\pi_{1}(C)=G
$$

We will assume that $G$ is finite ${ }^{1}$. If we consider a D-brane (of the appropriate dimension in the appropriate theory) wrapped around $C$ we obtain a particle in some supersymmetry multiplet, depending on the theory we are dealing with. However we have more degrees of freedom available to us: On the $\mathrm{D}$-brane worldvolume we have a $U(1)$ gauge symmetry. The vacuum configurations of the D-brane correspond to particles. However the existence of a non-trivial fundamental group introduces a new subtlety. The vacuum equation on the D-brane worldvolume implies

$$
F=0 .
$$

This equation can be solved in more than one way. The simplest way is to set $A=0$, but we can also set $A$ to be a non-trivial flat $U(1)$ connection. The number of inequivalent ways this can be done is equal to the number of inequivalent 1 dimensional representations of $G$. To see this, let $p_{\gamma}$ denote a one dimensional path in $C$ representing each $\gamma \in G$. Let

$$
g_{\gamma}=\exp \left(i \int_{p_{\gamma}} A\right)
$$

\footnotetext{
${ }^{1}$ The considerations of this paper can be easily generalized to the more general case where $G$ has isolated finite dimensional unitary representations.
} 
denote the wilson line for that path, where we are assuming $F=d A=0$. Note that due to flatness, this does not depend on which representative path we take. Then $g_{\gamma}$ satisfy the same relations as the fundamental group $G$ by composing the holonomies along the composed paths. In other words we can view $\left\{g_{\gamma}\right\}$ as forming a one dimensional representation of $G$. We thus conclude that the number of inequivalent $D$-brane states wrapped once around $C$ is equal to the number of one dimensional representations of $G .^{2}$ Note that, even though the argument about the ground states we gave is classical, the masses of the corresponding D-branes are protected quantum mechanically by the BPS condition. Thus the classical arguments suffice for the existence of these BPS ground states.

Now let us consider $N$ D-branes wrapped around $C$. Let us assume that the dynamics is such that without turning on gauge fields there are no multiwrapped bound states (as is the case for example for the conifold [4]). Now let us ask if there are any new bound states due to the existence of a nontrivial fundamental group on $C$. In this case we have a $U(N)$ gauge theory on the D-brane worldvolume. In solving the flatness condition $F=0$ we can, as in the case with a single D-brane, turn on non-trivial flat connections. For the same reason as before the number of inequivalent ways this can be done corresponds to the number of inequivalent $N$-dimensional representations of $G$. However, the reducible representations will not correspond to a bound state: The fact that we have reducible representation means that we can find at least one $U(1)$ subgroup of $U(N)$ which commutes with the turned on wilson lines. This in particular means that the scalars $\phi$ in that $U(1)$ multiplet are massless. This is because the $\mathrm{D}$-brane action which couples the fields to the background gauge field $A_{c}$ does so by group commutators

$$
S=\ldots+\int\left|\left[\phi, A_{c}\right]\right|^{2}
$$

and this is zero for the choice of $U(1)$ direction we have made. The massless scalar mode $\phi$ is responsible for seperating D-branes (dividing D-branes in some particular way). Thus the same arguments that shows that multiwrapped states are not bound, applied to this $U(1)$, show that the reducible representations do not give rise to bound states. Let us instead assume that $N=d_{i}$ where $d_{i}$ is the dimension of some irreducible representation of $G$. In this case we can identify the holonomies of the $U(N)$ gauge theory with this irreducible representation of $G$. The very fact that the representation is irreducible, means that no massless deformation exists, i.e. all commutators

\footnotetext{
${ }^{2}$ Note that if $C$ had a non-trivial $S^{1}$, then $G$ would have $\mathbf{Z}$ as a subgroup. In this case one does not get new bound states. This is because turning on Wilson lines around $S^{1}$ have a continuous parameter associated with it, and to find the bound states we need to find the ground states of the quantum mechanics problem on the dual circle. As is well known (apart from the usual fermionic degrees of freedom) this is one dimensional.
} 
of the form (2.1) are non-trivial and give mass to all such directions, except for the overall $U(1)$ which corresponds to the center of mass deformation. We thus automatically get a bound state. We conclude that the number of bound states are in one to one correspondence with the number of irreducible representations of $G$ which in turn is equal to the number of conjugacy classes of $G$. Moreover the dimension of the representation is the number of D-branes wrapped around $C$.

\subsection{Discrete Quantum Numbers and D-Brane/String Bound States}

One way one obtains a discrete non-trivial fundamental group is by modding a simply connected space by some free group action $G$. In this case the fundamental group of the space is $G$. If we are dealing with type II string theories compactified on such spaces, we get the familiar twisted sectors of strings, which correspond to strings which are closed up to the $G$ action. We get twisted sectors, one for each conjugacy class of $G$. Moreover the interaction between them is consistent with the group multiplication for all the elements in a conjugacy class. We can define a discrete symmetry which is consistent with this interaction (in orbifold constructions this is known as a 'quantum symmetry'): Let $\hat{G}$ be the abelianization of $G$ (i.e. $\hat{G}=G /[G, G])$. Then for each conjugacy class $[g] \in G$ we get an element of $\hat{G}$ (this is not a one to one map in the general case). Then the string interactions are consistent with the abelian multiplication of $\hat{G}$, and we can thus associate a $\hat{G}$ symmetry to strings. In the case $G$ is abelian, $\hat{G}=G$ and the symmetries of the orbifold theory we have are in fact the same as $G$. For example if $G=\mathbf{Z}_{n}$, in the orbifold theory we also get a new $\mathbf{Z}_{n}$ symmetry, which is simply the conservation law associated with the labels of the twisted sectors. This is reflected by associating a phase $\exp (2 \pi i k / n)$ to states coming from the $k$-th twisted sector. In fact we can summarize the general case by saying that the discrete symmetries of the orbifold theory correspond to choosing a representation of $\hat{G}$ which is the same as finding 1-dimensional representations of $G$.

It is natural to ask whether the wrapped D-branes we have discussed above carry charge under this $\hat{G}$. Let us first do this for the case of $G=\mathbf{Z}_{n}$. It is convenient to consider the case where $n \rightarrow \infty$, i.e. the case where the group is $\mathbf{Z}$ and view the other cases as embeddable in it. In particular let us consider $S^{1}=R / \mathbf{Z}$. In this case the corresponding notion of the twisted sectors are just the winding sectors, and the new $Z$ charge is just the winding number charge. Let us denote the winding direction by 1 . A winding string is charged under the $B_{01}$ field. Consider a D-brane which is wrapped around this circle. Then considering a winding number $n$ state of string, amounts to considering a state with electrical flux of $n$ units [3], i.e, 
$d A_{1} / d t=n$. Note that this is the conjugate variable to the wilson line $A_{1}$. Let us denote the Wilson lines by $A_{1}=\theta$. Then we can view a sector with a given Wilson line as a superposition of the electrical flux states (in the usual position/momentum representation):

$$
|\theta\rangle=\sum \exp (i n \theta)|n\rangle
$$

Indeed, in terms of a T-dual (in direction 1) picture, $\theta$ is the center of mass position and the winding $n$ goes over to the momentum around the dual circle. Thus the conservation law associated with the addition in the winding number, gets mapped to the shift symmetry in the $\theta$ angle which is the Wilson line on the D-brane world volume. In other words, the symmetry

$$
|n\rangle \rightarrow \exp (i n \alpha)|n\rangle
$$

gets mapped to

$$
|\theta\rangle \rightarrow|\theta+\alpha\rangle
$$

The $\mathbf{Z}_{n}$ version, which is the case of interest for us, is now clear: If we consider the symmetry which corresponds to multiplying the first $\mathbf{Z}_{n}$ twisted state of string by $\exp (2 \pi i / n)$ it acts on the D-brane world volume by turning on one unit of $\mathbf{Z}_{n}$ Wilson line. In other words it permutes the D-brane bound states. In order to get eigenstates of this action of the quantum $\mathbf{Z}_{n}$ charge operator, we can take a superposition of D-branes with discrete Wilson lines turned on. A natural question arises as to whether we can describe these superpositions in a classical way. In fact from what we said before this can be partially done, namely we can turn on discrete electrical fluxes which would correspond to these eigenstates. In fact we can also represent the electric fluxes as the twisted string states themselves. Thus the D-brane states with a superposition of different discrete Wilson lines can be viewed as bound states of D-branes without any Wilson line, with twisted strings. The actual ground state looks very different from a string attached to the D-brane-in particular it has the same mass, by the BPS condition, as the D-brane without the string attached. The ground state is a twisted string completely 'dissolved' in the D-brane.

This can be easily generalized to include the non-abelian case: Each Dbrane bound state is characterized by an irreducible representation of $G$. The action of a particular symmetry of $\hat{G}$, corresponding to a one dimensional representation $R_{1}$ of $G$, on the wrapped D-brane state corresponding to representation $R$ is given by

$$
R \rightarrow R \otimes R_{1}
$$

(This is a shift in the $U(1)$ part of the $U(N)$ holonomy - precisely the action of the generator of the $\hat{G}$ symmetry.) This in general permutes the corresponding D-brane bound states given by specific Wilson lines. Clearly we 
can choose a linear superposition of D-brane bound states to form eigenstates of this action, and again these will correspond to bound states of D-branes associated with the representation $R$ with a twisted string state, as in the abelian case.

\section{Examples Based on Calabi-Yau Threefold}

As a concrete application of the above ideas let us consider the case of type IIB compactification on Calabi-Yau threefolds, and let us consider the limit where the threefold develops a conifold singularity. This means that locally the manifold looks like

$$
z_{1} z_{4}-z_{2} z_{3}=\mu
$$

As $\mu \rightarrow 0$ the manifold is singular at $z_{1}=z_{2}=z_{3}=z_{4}=0$. In this example one has a three sphere which vanishes in this limit. One way to see the $S^{3}$ is to consider (assuming $\mu$ is real) the locus where $z_{4}=z_{1}^{*}$ and $z_{3}=-z_{2}^{*}$. To study this space in more detail we consider the $2 \times 2$ matrix

$$
A=\left(\begin{array}{ll}
z_{1} & z_{3} \\
z_{2} & z_{4}
\end{array}\right)
$$

In terms of this, the local model for the conifold is given by

$$
\operatorname{det}(A)=\mu
$$

There is an $S L(2, \mathbf{C})_{L} \times S L(2, \mathbf{C})_{R}$ acting on the conifold space by left and right multiplication of $A$ :

$$
A \rightarrow K_{L} A K_{R}
$$

If we consider the 3-brane wrapped around $S^{3}$, then we get a hypermultiplet charged under $U(1)$, with mass proportional to $\mu$ [1], where from the gauge theory viewpoint $\mu$ is the vev of the scalar in the $N=2 U(1)$ vector multiplet. As $\mu \rightarrow 0$ we obtain a massless hypermultiplet. One signature of the massless hypermultiplet is the fact that the coupling constant of the $U(1)$ near $\mu \rightarrow 0$ is given by $\tau=\frac{1}{2 \pi i} \log \mu$ which is what one expects from the 1-loop correction for $N=2$ QED with a hypermultiplet of mass $\mu$. Note that the coupling constant undergoes a monodromy $\tau \rightarrow \tau+1$ as $\mu \rightarrow \mu \exp (2 \pi i)$. In other words the $\theta$ angle shifts by $2 \pi$. This in particular means that the magnetic states of charge one become dyonic as we go around the origin in $\mu$ space. This is in fact the Witten effect [5]. Geometrically this is realized rather nicely by the fact that a magnetic state of charge one is represented by a 3-cycle which intersects this $S^{3}$ at one point. Let us call this class $[b]$. Moreover let ue denote the class of the $S^{3}$ cycle by $[a]$. Then as $\mu$ goes 
around the origin we come back to the same geometry but the cycles undergo the monodromy

$$
[b] \rightarrow[b]+[a]
$$

Thus the magnetic state picks up one unit of electric charge.

We now consider modding out this space by a discrete subgroup of $S U(2)$. There are a number of ways this can be done depending on how one embeds $S U(2)$ in the $S L(2, \mathbf{C})_{L} \times S L(2, \mathbf{C})_{R}$. For the purposes of this paper, it suffices to consider the $S U(2)$ identified with the $S U(2)$ subgroup of $S L(2, \mathbf{C})_{L}$. Let $G$ denote a discrete subgroup of $S U(2)$. Then modding out the conifold by $G$ :

$$
A \rightarrow G \cdot A
$$

gives rise to a new space, which again satisfies the Calabi-Yau condition. Note that this action is free, i.e., we have no fixed points. This in particular means that the new space will have a fundamental group isomorphic to $G$. Note also that the $S^{3}$ is mapped to itself under the $G$ action. Now consider the 3-cycle

$$
C=S^{3} / G
$$

Note that $\pi_{1}(C)=G$. Now the situation is similar to what we had before modding out, except that the minimal three cycle charge is now given by a 3-brane wrapped $1 /|G|$ times around the $S^{3}$, where $|G|$ denotes the order of $G$. In particular the basic unit of charge is reduced by a factor of $1 /|G|$. Now we will apply the considerations of the previous section and deduce the bound state spectrum of the D3 branes wrapped around $C$.

In order to do this, it is convenient to first review some basic facts about the $S U(2)$ subgroups. They are in one to one correspondence with A-D-E. The cyclic subgroup of $S U(2)$ is identified with the A series, the Dihedral subgroup with $\mathrm{D}$ and the exceptional subgroups with the $\mathrm{E}$ series (this is familiar from how the gauge symmetries arise in modding out $\mathbf{C}^{2}$ by the corresponding discrete subgroup of $S U(2))$. This correspondence has an interesting further aspect: If we consider the affine Dynkin diagram associated with each subgroup $G$ of $S U(2)$, the number of nodes (which is equal to the rank of the corresponding Lie group plus one) is equal to the number of irreducible representations of $G$. Moreover the dimension of the corresponding representation is in 1-1 correspondence with the Dynkin index associated with that node (see figure 1).

In fact one can also deduce the tensor product of the fundamental representation of $S U(2)$ with any of the other representations from Dynkin diagrams: Tensoring the fundamental representation with an irrep corresponding to a node yields the direct sum of the irreps associated with all the adjacent nodes. 

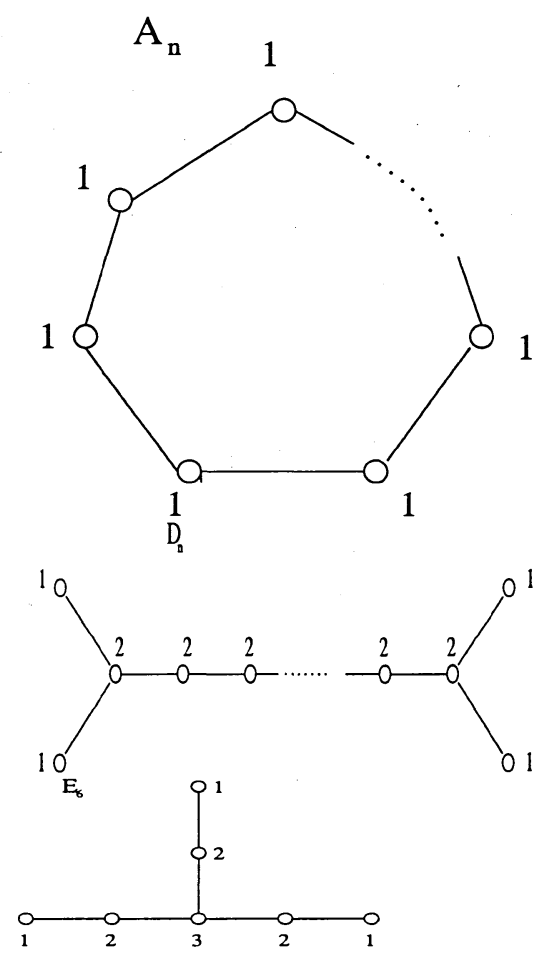

$E_{\text {, }}$
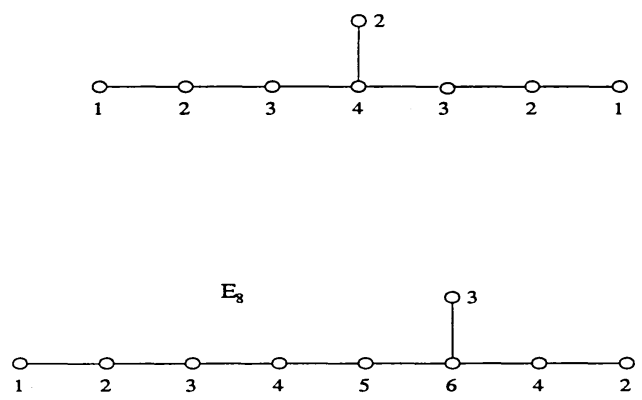

Figure 1: The A-D-E Affine Dynkin diagrams encode the information about the representations of cyclic, dihedral and exceptional subgroups of $S U(2)$. Each node is associated with an irreducible representation, and the Dynkin index corresponds to the dimension of the representation. 
Coming back to our problem of finding the number of D3-brane states wrapped around $C$, using the result of the previous section we see that they are in one to one correspondence with the irreps of $G$ which are in turn in one to one correspondence with the nodes of the corresponding affine A-D-E, with the Dynkin index denoting the number of D3 branes wrapped around $C$. Note that this number is also the same as the $U(1)$ charge of the modded theory (the minimal charge is $1 /|G|$ times the minimal $U(1)$ charge of the unmodded theory). Also note that the BPS condition implies that their mass is given by $M_{i}=\mu d_{i} /|G|$, where $d_{i}$ denote the Dynkin indices.

\subsection{Various Checks of the Results}

We now pass the previous results through some checks. First let us ask what should the correction be to the effective coupling of the $U(1)$ theory, normalized so that the basic electric unit is $1 /|G|$ times the unmodded theory. To see that it suffices to see how a magnetic state will undergo monodromy as $\mu$ goes around the origin. The magnetic state, given by the cycle $[b]$ in the unmodded theory, continues to be the minimal unit in the modded theory as well (because it intersect the $S^{3} / G$ at one point). Thus the same monodromy continues to hold, namely $[b] \rightarrow[b]+[a]$ but now $[a]$, which is the $S^{3}$ class, does not represent the minimal charge state for the new $U(1)$ but $|G|$ times the minimal unit. Thus the $\theta$ angle for the new $U(1)$ undergoes a monodromy $2 \pi|G|$. This should be reproducible as a result of the contribution of the light modes. In the above we have argued that there are as many light modes as the nodes of the corresponding affine Dynkin diagram and that each will carry a charge $q_{i}=d_{i}$ which is the same as the Dynkin index associated with the corresponding node, with mass $M_{i}=\mu d_{i} /|G|$. The contribution they make to the coupling constant of the $U(1)$ theory is given by the usual one loop result,

$$
\tilde{\tau}=\sum_{i} q_{i}^{2} \log \mathrm{M}_{\mathrm{i}}=\left(\sum q_{i}^{2}\right) \log \mu+\text { const }
$$

Thus we pick up the monodromy, as $\mu$ goes around the origin, given by

$$
\sum q_{i}^{2}=\sum d_{i}^{2}
$$

We expected this to be equal to $|G|$, and this is indeed the case:

$$
\sum d_{i}^{2}=|G|
$$

and expresses the well known relation between the dimension $d_{i}$ of irreducible representation of a group $G$ with its order $|G|$. We find this a strong check on our basic conclusion. 
Let us consider another check. One of the basic aspects of the existence of massless modes corresponding to wrapped D3 branes around vanishing 3 -cycles is that it provides a way to go to new Calabi-Yau compactifications by giving vevs to them [6]. If we consider the case where an $S^{3}$ shrinks to zero size, locally we can consider growing an $S^{2}$ instead. This is described as follows. Consider the point where $\mu=0$. In such a case we can define a complex parameter $\lambda=\lambda_{1} / \lambda_{2}$ through the equation

$$
\left(\begin{array}{ll}
z_{1} & z_{3} \\
z_{2} & z_{4}
\end{array}\right)\left(\begin{array}{l}
\lambda_{1} \\
\lambda_{2}
\end{array}\right)=0
$$

Note in particular that the point $z_{1}=z_{2}=z_{3}=z_{4}=0$ which was singular is now replaced by the sphere parameterized by $\lambda$, i.e., we have blown it up to an $S^{2}$. We can use $z_{1}, z_{2}, \lambda$ as parametrizing the blown up space (note that $z_{3}=-\lambda z_{1} \quad z_{4}=-\lambda z_{2}$ ). The size of $S^{2}$ should be described by varying the vev of a hypermultiplet, and that is precisely identified with the massless hypermultiplet coming from the wrapped D3 brane around the vanishing $S^{3}$. In the compact set up there are some global obstructions as to the sizes of the $S^{2}$ 's coming from D-term constraints in field theory (see [7] for the relation of these constraints to a geometric realization of confinement); however even in such cases the existence in the field theory of a degree of freedom which describes the geometric size of blown up $S^{2}$ is a check of the fact that a D3 brane wrapped around a vanishing $S^{3}$ corresponds to a massless hypermultiplet.

Now let us run this check in the case we are considering ${ }^{3}$ : If we mod out the conifold by $G$, this acts also on the blown up space. It is easy to see that the action of $G$ on $\lambda$ is trivial, and that as before $\left(z_{1}, z_{2}\right)$ form a doublet under the $G$ action. So for the blown up space given by $\left(z_{1}, z_{2}, \lambda\right)$ the ADE subgroups of $S U(2)$ just act on the two coordinates $\left(z_{1}, z_{2}\right)$. Thus the geometry we are getting is an ADE type singularity, parameterized by a sphere $\lambda$. The number of blow up modes in this case is familiar from the study of the corresponding ALE singularities and is given by the rank of the corresponding ADE, together with the mode corresponding to the size of the $S^{2}$ parametrized by $\lambda$. Thus we expect that the number of massless hypermultiplets be the rank of the corresponding ADE plus one. This is exactly what we found by the D-brane analysis. ${ }^{4}$ Note that this is the same as compactification of type IIB near an ADE singularity over an $S^{2}$, down to four dimensions. In this case one gets tensionless strings when the spheres

\footnotetext{
${ }^{3}$ We would like to thank Eric Zaslow for providing us with this blow up construction.

${ }^{4}$ Once we compactify on an extra circle, and go to the dual circle we end up with the type IIA description on the same manifold which leads to $N=4 \mathrm{ADE}$ gauge symmetries in 3 dimensions. In fact this may have interesting application for $3 \mathrm{~d} N=4$ mirror symmetries [8]along the lines suggested in [9].
} 
shrink [10]. However sometimes tensionless strings can look like ordinary string associated to higgsing of $U(1)$ factors [7] and this is indeed the case here.

Finally we can pass this through a string one loop test corresponding to the coefficient of $R^{2}$ term generated [11]. The coefficient of this term should be given by

$$
F_{1}=\sum_{i}-\frac{1}{12} \log M_{i}
$$

for hypermultiplets of masses $M_{i}$. This can be computed using the one loop topological string amplitudes [12]. There are only two cases where this has been computed ${ }^{5}$ with a singularity of the type we are considering. One corresponds to the case when one has a vanishing $S^{3}$ with a free $Z_{5}$ action [13], and in this case we expect to get a coefficient of $-\frac{5}{12} \log [\mu / 5]$ and this is indeed the case. And the other [14] (coming from the $Z_{3}$ quotient of a bicubic in $\mathbf{P}^{5}$ ) has an $S^{3} / Z_{3}$ shrinking and this gives the expected $-\frac{3}{12} \log [\mu / 3]$ contribution to $R^{2}$ term.

\subsection{Non-trivial Fundamental Group and Massless Wrapped $(p, q)$ Strings?}

Apart from the 3-branes that are wrapped around the shrinking 3-cycle in the case we have discussed so far, there are extra states that seem to be becoming light. These are $(p, q)$ strings of the IIB theory wrapped on any of the non-contractible loops of $S^{3} / G$. We cannot apriori argue that they are all stable. However we can use the discrete charges available to argue some may be stable. In particular for the fundamental strings, if we choose them to be the ground states corresponding to particular discrete symmetry $\hat{G}$, we can show to all order in string perturbation theory that they are stable. However, the wrapped $(p, q)$ string states, for weak string coupling, are very massive and no quantum charge protects their decay to fundamental string states. The only quantum charge they could carry is that of the discrete symmetry we have already discussed, and the light fundamental string states already carry them.

So let us concentrate on the wrapped fundamental string states and consider the limit where the $S^{3} / G$ shrinks. We can ask if they do give rise to additional massless stable states. First we should note that our intuition about their becoming massless is purely at a classical level. Quantum mechanically we must see if a BPS mass formula protects their mass. However in this case, the strings are not charged under the Ramond-Ramond gauge field that couples to the 3-brane. The only charge they carry is under a discrete gauge group corresponding to the abelianisation of the fundamental

\footnotetext{
${ }^{5}$ We are grateful to Albrecht Klemm for pointing these out to us.
} 
group, as noted before, and this does not give rise to any BPS mass formula. At any rate classically one would naively expect that one ends up with stable massless states as the $S^{3} / G$ shrinks. We do not believe this happens, for the following reason: Even if we assume the naive classical mass for them is valid, the physics of the light modes is dominated by the light wrapped 3 -branes whose mass is proportional to the volume of the 3-cycle. The light strings have classical masses which are proportional to $1 / 3$ power of the volume and thus are much heavier in the limit the volume shrink. Moreover even though the ground states of the fundamental string states in the twisted sectors are protected from decay to all orders in string perturbation theory by the discrete charge they carry, this is not the case non-perturbatively, because the wrapped D3 branes not only are lighter but also carry the same discrete charges, as discussed before. Thus a fundamental string can decay to a pair of D3 and anti-D3 branes which are wrapped (with suitable Wilson lines turned on). This seems to provide an interesting example for perturbative string states which are stable to all order in string perturbation, but nevertheless become unstable in the regime where string perturbation is not valid (where the $S^{3} / G$ shrinks).

\section{5-Branes}

Up to now we have talked about D-branes wrapped around cycles with nontrivial $\pi_{1}$. Can we do a similar thing for NS 5-branes of type IIA or M5 brane of M-theory, and NS 5-brane of type IIB?

For type IIB NS 5-brane the story is the same as what we have said for D-branes, because there is a gauge field $A$ living on the worldvolume (after all it is S-dual to D5 branes).

The story is more subtle for NS 5-branes (or M5 branes) of type IIA (Mtheory). For a single 5-brane, given that we have the anti-symmetric tensor field in its world volume, the question of choosing non-trivial discrete vev's is exactly the same as the choice of a discrete torsion [15]. In particular if the spatial part is wrapped around the 5-cycle $C$, the inequivalent choices for the vev of $B_{i j}$ correspond to the choice of $H^{2}(C, U(1))$. The more subtle question is whether there is anything new that happens when we have more than one 5 -brane. In this case there is no classical analog for a non-abelian object such as $\pi_{1}$ (note for example that $\pi_{2}$ is always abelian). However we will argue, at least for certain cases, that quantum mechanically there are additional choices available. Consider in particular the 5 dimensional submanifold to be $C=S^{1} \times \tilde{C}$ and suppose that $\pi_{1}(\tilde{C})$ is a finite non-abelian group. In this case for each $n$ dimensional representation of $\pi_{1}(\tilde{C})$, if we consider $n 5$-branes wrapped around $C$ we have new bound states corresponding to turning on certain fields. To see this we note that $n 5$-branes wrapped around $S^{1}$ give 
rise to $U(n)$ gauge theory in $4+1$ dimensions. Thus on $\tilde{C}$ we can turn on non-abelian wilson line, as we did for the D-brane case in this paper. This example shows that even for 5-branes of type IIA (or M-theory) there are in general non-abelian version of wilson line. It would be interesting to see what is the more general version of this non-abelian vev, when we do not assume that $C$ has an $S^{1}$ factor.

We would like to thank A. Klemm, J. Maldacena, A.Strominger and E. Zaslow for valuable discussions.

The research of R.G. was supported by DOE grant 91-ER40618. The research of C.V. was supported in part by NSF grant PHY-92-18167.

\section{References}

[1] A. Strominger, Nucl. Phys. B451 (1995) 96.

[2] J. Polchinski, Phys. Rev. Lett. 75 (1995) 4724.

[3] E. Witten, Nucl. Phys. B460 (1996) 335.

[4] M. Bershadsky, V. Sadov, and C. Vafa, Nucl. Phys. B463 (1996) 420.

[5] E. Witten, Phys. Lett. B86 (1979) 283.

[6] B. Greene, D. Morrison, and A. Strominger, Nucl. Phys. B451 (1995) 109.

[7] B. Greene, D. Morrison, and C. Vafa, Nucl. Phys. B481 (1996) 513.

[8] K. Intriligator and N. Seiberg, Phys. Lett. B387 (1996) 513.

[9] K. Hori, H. Ooguri, and C. Vafa, Nucl. Phys. B504 (1997) 147.

[10] E. Witten, "Comments on String Dynamics", "Proceedings of Strings $95 "$.

[11] C. Vafa, Nucl. Phys. B447 (1995) 252.

[12] M. Bershadsky, S. Cecotti, H. Ooguri, and C. Vafa, Comm. Math. Phys. 165 (1994) 311.

[13] P. Aspinwall and D. Morrison, Phys. Lett. B334 (1994) 79.

[14] A. Klemm, unpublished.

[15] C. Vafa, Nucl. Phys. B273 (1986) 592. 